\title{
Grain size, size-distribution and dislocation structure from diffraction peak profile analysis
}

\author{
T. Ungár and J. Gubicza \\ Department of General Physics, Eötvös University Budapest, H-1518, P.O.B 32, Budapest, \\ Hungary
}

\begin{abstract}
Diffraction peak profile analysis (or Line Profile Analysis, LPA) has recently been developed to such an extent that it can be applied as a powerful method for the characterization of microstructures of crystalline materials in terms of crystallite size-distribution and dislocation structures. Physically based theoretical functions and their Fourier coefficients are available for both, the size and the strain diffraction profiles. Strain anisotropy is rationalized in terms of the contrast factors of dislocations. The Fourier coefficients of whole diffraction profiles are fitted by varying the following fundamental parameters characterizing the microstructure: (i) $m$ and (ii) $\sigma$, the median and the variance of the log-normal size distribution function, (iii) $\rho$ and (iv) $M$, the density and the arrangement parameter of dislocations and (v) $q$ or $q_{1}$ and $q_{2}$ for the average dislocation contrast factors in cubic or hexagonal materials, respectively. The method will be illustrated by showing results on ECA pressed copper and titanium.
\end{abstract}

Keywords: crystallite-size, size-distrubution, dislocation-density, slip-geometry

\section{Introduction}

Grains, subgrains or dislocation cells and the dislocation structure in crystalline materials can directly be observed in micrographs of transmission electron microscopy (TEM). These observations provide details on the micron or submicron scales. Average properties of the microstructure, especially on the hundreds of microns up to millimeters scale like crystallite size-distributions [1-3] or specific properties of dislocations as long range internal stresses [47], dislocation densities [8-10], arrangement parameters [11,12] or statistical fluctuations [13$15]$ are better obtained by diffraction peak profile analysis [16].

Diffraction peaks broaden when crystallites are small or the material contains lattice defects. The diffraction order dependence of the two effects is different enabling their separation. The two classical methods for this are: (i) the Williamson-Hall [17] and (ii) the Warren-Averbach [18] procedures. The first is based on the Full Widths at Half Maximum (FWHM) or the integral breadths while the second on the Fourier coefficients of diffraction profiles. Both methods provide apparent size parameters of coherently scattering domains and values of the mean square strain. Strain anisotropy [19] and sometimes shape anisotropy [20] make the evaluation complicated. Strain anisotropy means that neither the breadth nor the Fourier coefficients of the diffraction profiles are monotonous functions of the diffraction angle or $g$ where $g$ is the absolute value of the diffraction vector [18-27]. Further difficulty is encountered by the fact that the mean square strain, $\left\langle\varepsilon_{L, g}{ }^{2}>\right.$, is never a constant, neither as a function of $L$ nor $g$, where $L$ is the Fourier length [2,5-16,18,21-24]. In order to separate strain and size broadening correctly strain anisotropy has to be treated properly. Two different models have been developed so far: (i) the phenomenological model based on the elastic anisotropy of crystals [27] and (ii) the dislocation model [22] based on the mean square strain of dislocated 
crystals $[8,9,11,12]$. Latter takes into account that the effect of dislocations on strain broadening depends on the relative orientations of the line and Burgers vectors of dislocations and the diffraction vector, similarly as the contrast effect of dislocations in electron microscopy. Anisotropic contrast can be summarised in contrast factors, $C$, which can be calculated numerically on the basis of the crystallography of dislocations and the elastic constants of the crystal [11,21-24,28]. Using the average contrast factors the modified Williamson-Hall and the modified Warren-Averbach procedures have been suggested [22], enabling to determine (i) different averages of crystallite sizes, (ii) the density and (iii) the effective outer cut off radius of dislocations $[22,29]$. A method has recently been developed to determine (a) crystallite size distribution and the dislocation structure in terms of (b) density, (c) arrangement parameter and (d) character of dislocations [29,30]. In the present work diffraction peak profile analysis is outlined briefly and applied to characterise the size distribution of crystallites and the dislocation structure in submicron grain size copper and titanium.

\section{Evaluation of broadened diffraction peak profiles}

\subsection{Fundamental equations}

In the kinematical theory of diffraction the Fourier coefficients of the physical profiles of Bragg reflections can be given by the Warren-Averbach equation [18]:

$$
A_{L}=A_{L}^{S} A_{L}^{D}=A_{L}^{S} \exp \left[-2 \pi^{2} L^{2} g^{2}<\varepsilon_{g, L}^{2}>\right]
$$

where $L$ is the Fourier variable: $L=\mathrm{n} a_{3}, \mathrm{n}$ are integers and $a_{3}$ is the unit of the Fourier length in the direction of the diffraction vector $g: a_{3}=\lambda /\left[2\left(\sin \theta_{2}-\sin \theta_{1}\right)\right]$, the diffraction profile is mesured in the angular range from $\theta_{1}$ to $\theta_{2}$ and $\lambda$ is the wavelength of the X-rays (or neutrons). In dislocated crystals the mean square strain is $[11,12]$ :

$$
<\varepsilon_{g, L}^{2}>=-(b / 2 \pi)^{2} \pi \rho C f(\eta),
$$

where $\rho$ is the dislocation density, $b$ and $C$ are the Burgers vector and the contrast factor of dislocations, respectively, $\eta=L / \mathrm{R}_{\mathrm{e}}, \mathrm{R}_{\mathrm{e}}$ is the effective outer cut-off radius of dislocations and $f(\eta)$ is a function derived explicitely by Wilkens for dislocations, see equations A.6 to A.8 in [12] or eqs. (22) and (23) in [29]. For small values of $\eta$ the Wilkens function can be approximated by a logarithmic function $[6,11,12]$ :

$$
<\varepsilon_{g, L}^{2}>\cong \frac{\rho C b^{2}}{4 \pi} \ln \left(\mathrm{R}_{\mathrm{e}} / L\right)
$$

Inserting (3) into the Warren-Averbach equation in (1) the modified Warren-Averbach equation can be obtained [22]:

$$
\ln A(\mathrm{~L}) \cong \ln A^{\mathrm{S}}(\mathrm{L})-\rho \mathrm{BL}^{2} \ln \left(\mathrm{R}_{\mathrm{e}} / \mathrm{L}\right)\left(K^{2} \bar{C}\right)+O\left(K^{4} \bar{C}^{2}\right) \text {, }
$$

$O$ stands for higher order terms in $K^{2} \bar{C}$. The full width at half maximum (FWHM) or the integral breadths of profiles can be plotted versus $K=2 \sin \theta / \lambda$ (where $\theta$ is the diffraction angle and $\lambda$ is the wavelength of X-rays or neutrons) in the classical Williamson-Hall plot [17]. The intercepts and the slopes of the regressions through the mesured data should provide apparent size parameters and values of the mean square strain, respectively. Due to strain anisotropy, however, the data points usually do not follow smoth curves making reliable regressions 
impossible. It can be shown that the anisotropic contrast of dislocations enables the rationalisation of strain anisotropy in terms of the modified Williamson-Hall plot [22]:

$$
\Delta K \cong \alpha / \mathrm{D}+\left(\pi T b^{2} / 2\right) \rho^{1 / 2} K^{2} \bar{C}+O\left(K^{4} \bar{C}^{2}\right),
$$

where $\Delta K$ is either the FWHM or the integral breadth of profiles, $D$ is the so called apparent size parameter, $\alpha$ is 0.9 or 1 for the FWHM or the integral breadth, respectively, and $T$ is a constant depending on the effective outer cut-off radius of dislocations [22].

\subsection{The contrast factors of dislocations}

In a texture free cubic or hexagonal polycrystal or if the Burgers vector population on the different slip systems is random the contrast factors of dislocations $C$ can be averaged over the permutations of the $h k l$ indices and the so called averaged contrast factors, $\bar{C}$, are [31]:

$$
\bar{C}=\bar{C}_{h 00}\left(1-q H^{2}\right),
$$

or

$$
\bar{C}=\bar{C}_{h k 0}\left[1+\frac{\left[A\left(h^{2}+k^{2}+(h+k)^{2}\right)+B l^{2}\right] l^{2}}{\left[h^{2}+k^{2}+(h+k)^{2}+\frac{3}{2}\left(\frac{a}{c}\right)^{2} l^{2}\right]^{2}}\right],
$$

respectively, where $\bar{C}_{h 00}$ and $\bar{C}_{h k 0}$ are the average dislocation contrast factors for the $h 00$ and $h k 0$ reflections, respectively, $H^{2}=\left(h^{2} k^{2}+h^{2} l^{2}+k^{2} l^{2}\right) /\left(h^{2}+k^{2}+l^{2}\right)^{2} ; q, A$ and $B$ are parameters depending on the elastic constants and on the character of dislocations (e.g. edge or screw type) in the crystal and $a$ and $c$ are the two lattice constants in the hexagonal crystal.

The expression (7) can be rewritten as [33]:

$$
\bar{C}=\bar{C}_{h k 0}\left[1+q_{1} \mathrm{x}+q_{2} \mathrm{x}^{2}\right],
$$

where

$$
\mathrm{x}=\frac{2}{3 a^{2}}\left(\frac{l}{g}\right)^{2}
$$

$g$ is the absolute value of the diffraction vector and $q_{1}=A$ and $q_{2}=B-(3 / 2) A(a / c)^{2}[33]$.

\subsection{The size Fourier coefficients}

Log-normal is one of the most commonly used size distribution density function:

$$
f(x)=\frac{1}{\sqrt{2 \pi} \sigma} \frac{1}{x} \exp \left\{-\frac{[\ln (x / m)]^{2}}{2 \sigma^{2}}\right\},
$$


where $\mathrm{x}$ is the grain or crystallite size and $\sigma$ and $\mathrm{m}$ are the variance and the median of the size distribution function, respectively. Assuming spherical crystallite shape the size Fourier coefficients in eq. (1) can written as [29,30]:

$$
\begin{aligned}
& A^{\mathrm{S}}(\mathrm{L}) \sim \frac{m^{3} \exp \left(4.5 \sigma^{2}\right)}{3} \operatorname{erfc}\left[\frac{\log (|L| / m)}{\sqrt{2} \sigma}-1.5 \sqrt{2} \sigma\right]- \\
& -\frac{m^{2} \exp \left(2 \sigma^{2}\right)|L|}{2} \operatorname{erfc}\left[\frac{\log (|L| / m)}{\sqrt{2} \sigma}-\sqrt{2} \sigma\right]+\frac{|L|^{3}}{6} \operatorname{erfc}\left[\frac{\log (|L| / m)}{\sqrt{2} \sigma}\right],
\end{aligned}
$$

where $\operatorname{erfc}$ is the complementary error function. Hinds has shown that the area-, volume- and arithmetically weighted mean crystallite sizes can be obtained from $m$ and $\sigma$ in a straightforward manner [32]:

$$
\begin{aligned}
& <\mathrm{x}>_{\text {area }}=\mathrm{m} \exp \left(2.5 \sigma^{2}\right), \\
& <\mathrm{x}>_{\mathrm{vol}}=\mathrm{m} \exp \left(3.5 \sigma^{2}\right), \\
& <\mathrm{x}>_{\text {arithm }}=\mathrm{m} \exp \left(0.5 \sigma^{2}\right) .
\end{aligned}
$$

Here we make the following note on the interpretation of crystallite size as determined by Xrays. Size broadening is caused by the column length of coherently scattering domains where this length is parallel to the diffraction vector. As stressed above, an assumption is made first on the shape and on the size distribution of the coherently scattering domains. In the present case the crystallite shape is considered as spherical and the crystallite sizes are supposed to fulfil a log-normal distribution. Hence the average diameters and the parameters of the size distribution function can be determined. These parameters, especially the average diameter, need not be identical with other length scales such as for example, the grain size or the particle size provided by scanning or transmission electron microscopy (SEM or TEM). Coherently scattering domain means the region wherein the amplitudes of the scattered X-rays sum up. When the crystallographic orientation between regions exceed a few degrees the amplitude summation ends and intensities are summing up. This means that the X-ray crystallite size corresponds to domains or regions wherein the variations of orientations are smaller than a few degrees. This type of regions may belong to the same grain or particle in a SEM or TEM micrograph. It is important to note, however, that single dislocations do not disturb the coherency of X-ray scattering since the misorientation they cause is of the order of $b \rho^{1 / 2}$. Taking typical values for $b$ and $\rho$ in plastically deformed copper, $0.26 \mathrm{~nm}$ and $1 \times 10^{15} \mathrm{~m}^{-2}$, this misorientation is of the order of $\sim 0.5^{0}$. Special arrays or bundles of dislocations can easily create misorientations of a few degrees thus creating boundaries of the coherently scattering regions. There may be some sort of proportionality between the X-ray crystallite size and the grain or particle size determined by SEM or TEM. However, to the best knowledge of the authors this has not yet been studied and goes beyond the scope of this work. From these considerations it is concluded thereafter that: (i) the dislocation density (or, in other words, microstrain) is a microstructural parameter independent of crystallite size (domain size) and neither can be deduced from each other, (ii) the X-ray crystallite size can never be larger than the grain or particle size obtained by SEM or TEM.

\subsection{The fitting procedure [30]}

A numerical procedure has been worked out for fitting the Fourier coefficients of the experimentally determined physical profiles by the Fourier coefficients of the theoretical size 
and strain functions as given in eqs. (1), (2), (6),(7) and (11), respectively. The measured profiles must first be corrected for background, instrumental effects and overlapping peaks. The numerical procedure is as follows: i) the Fourier coefficients of the measured physical profiles are computed making the instrumental correction by using the Stokes method, ii) the Fourier coefficients of the size and strain profiles are calculated by using eqs. (1) and (2) and (11), (iii) the calculated Fourier coefficients are fitted to the experimental values using the nonlinear Marquardt-Levenberg least squares procedure (Gnuplot program package under Unix system). The program for the fitting procedure is available on the web: http//www.renyi.hu/mwp, for more details see [30]. The following five or six fitting parameters are used: (i) $m$ and (ii) $\sigma$, the median and the variance of the log-normal size distribution density function in the size profile, (iii) $\rho$ and (iv) $M$, the density and the arrangement parameter of dislocations in the strain profile and (v) $q$ or $q_{1}$ and $q_{2}$ for the average dislocation contrast factors as in eqs. (6) or (7) for cubic or hexagonal crystals, respectively. The values of $\bar{C}_{h 00}$ or $\bar{C}_{h k 0}$ are not fitting parameters since they are only multiplicative factors in the Fourier coefficients of the strain profiles. From eqs. (1) and (2) it can be seen that the value of $\rho$ can only be determined if the values of $\bar{C}_{h 00}$ or $\bar{C}_{h k 0}$ are known. The values of $q$ have been compiled in [28] for different dislocation types and elastic constants in cubic crystals. A similar compilation will be available for hexagonal crystals in [33]. A typical example of the fitting of the Fourier coefficients of the measured physical profiles (open circles) by the theoretical Fourier transforms (solid lines), where latter are given in eqs. (1), (2) and (11) together with the contrast factors defined in eq. (7), are shown in Fig. 1. for ECA pressed titanium.

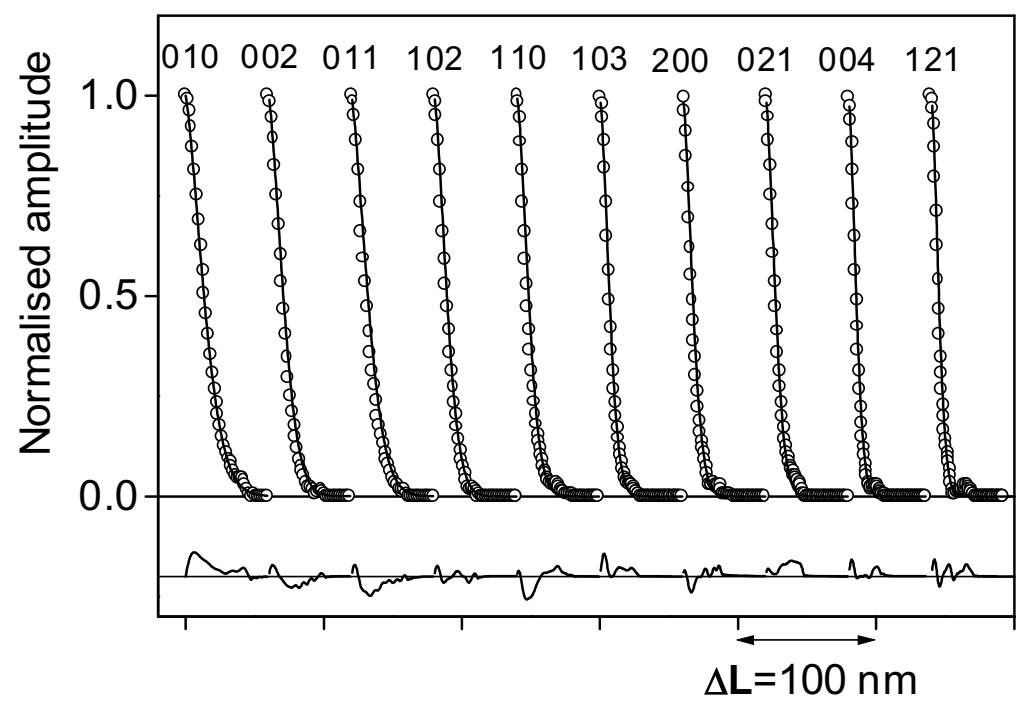

Figure. 1: The measured and fitted Fourier coefficients normalised to unity of ECA pressed titanium. The differences between the measured and fitted values are also shown in the lower part of the figure. The scaling of the differences is the same as in the main part of the figure. The indices of the reflections are also indicated.

\section{Experimentals}

\subsection{Samples}

A copper and a titanium sample having cubic and hexagonal crystal structure, respectively, were investigated. A 99.98\% copper and titanium specimens (kindly provided by Professor R. Valiev and Prof. Y. T. Zhu) were deformed by ECA (equal-channel angular pressing) producing submicron average crystallite size $[34,35]$. In order to avoid machining effects an approximately $100 \mu \mathrm{m}$ surface layer was removed from the specimen surface by chemical 
etching before the X-ray experiments.

\subsection{X-ray diffraction technique}

The diffraction profiles for copper were measured by a special double crystal diffractometer with negligible instrumental broadening (Wilkens \& Eckert, 1964). A fine focus rotating cobalt anode (Nonius FR 591) was operated as a line focus at $36 \mathrm{kV}$ and $50 \mathrm{~mA}(\lambda=0.1789 \mathrm{~nm})$. The symmetrical 220 reflection of a Ge monochromator was used in order to have wavelength compensation at the position of the detector. The $\mathrm{K} \alpha_{2}$ component of the Co radiation was eliminated by an $0.16 \mathrm{~mm}$ slit between the source and the Ge crystal. The profiles were registered by a linear position sensitive gas flow detector, OED 50 Braun, Munich. In order to avoid air scattering and absorption the distance between the specimen and the detector was overbridged by an evacuated tube closed by mylar windows.

The diffractogram for titanium specimen was measured by a Philips X'pert diffractometer using $\mathrm{Cu}$ anode and pyrolitic graphite secondary monochromator. The instrumental peak broadening was corrected by the measurement of the Si standard (NBS 640) using the Stokes method [36].

\subsection{Transmission electron microscopy}

Transmission electron microscopy (TEM, JEOL JEM200CX) has been used for direct measurement of the size distribution of crystallites in copper sample. Bright field images were used to measure the crystallite size in the samples.

\section{Results and discussions}

\subsection{The size distribution of grains or subgrains and the dislocation structure in ECA pressed copper}

The median, $m$, and variance, $\sigma$, of the crystallite size distribution, the dislocation densitiy, $\rho$, the arrangement parameter of dislocations, $M$, obtained for copper are listed in Table 1. It can be established that nanocrystalline materials was formed due to large deformation. The crystallite size distribution density function obtained from $m$ and $\sigma$ is plotted as a solid line in Fig. 2. For the $q$ parameter in the contrast factors $1.90 \pm 0.03$ has been obtained. In a previous work the values of $q$ have been calculated for the most common dislocation slip system in copper with the Burgers vector $\mathbf{b}=\mathrm{a} / 2<110>$ [28]. It was found that for pure screw or pure edge dislocations the values of $q$ are 2.37 or 1.68 , respectively. The experimental value obtained in the present case is somewhat below the arithmetic average of the two limiting values of $q$. From this we conclude that the character of the prevailing dislocations is more edge than screw. This is in good agreement with previous theoretical and experimental observations which has revealed that in fcc metals during large deformations at low temperatures screw dislocations annihilate more effectively then edge dislocations [37].

The crystallite size distribution obtained from X-rays is compared with size distribution determined from TEM micrographs of the plastically deformed bulk copper specimen. In the TEM micrographs the crystallite sizes were determined by the usual method of random line section. As a first approximation the grains, which are in contrast, were selected and the sizes of them were measured in the micrographs. The distribution of these sizes is shown in Fig. 2 by open squares. As it can be seen the grain sizes obtained from TEM are considerably larger than the sizes of crystallites (coherently scattering domains) determined from X-rays. A more careful evaluation of the magnified TEM micrographs reveals that the grains can be divided into smaller subgrains. The size distribution corresponding to the subgrains is shown as a bar 
graph in Fig. 2. The size distribution of subgrains is close to that of crystallites, however a small difference can be established in Fig.2. In the copper specimen investigated here the average dislocation distance is $\rho^{-1 / 2}=36 \mathrm{~nm}$. The volume and the area weighted mean crystallite size values (see eqs. (12) and (13)) are: 147 and $113 \mathrm{~nm}$, respectively. These crystallite size values are 3-4 times larger than the average dislocation distance indicating that the coherent domain size is definitely different from the dislocation distance. These experimental observations are in good correlation with the theoretical considerations in paragraph 2.3.

Table 1. The median, $m$, and the variance, $\sigma$, of the crystallite size distribution functions, the densities, $\rho$, and the arrangement parameters, $M$, of dislocations and the parameters of the dislocation contrast factors, $q$, or $q_{1}$ and $q_{2}$, obtained for copper and titanium by X-ray diffraction peak profile analysis.

\begin{tabular}{c|c|c|c|c|c|c} 
sample & $\mathrm{m}[\mathrm{nm}]$ & $\sigma$ & $\rho\left[10^{15} \mathrm{~m}^{-2}\right]$ & $M$ & $q$ & $q_{1} ; q_{2}$ \\
\hline \hline copper & $59 \pm 5$ & $0.51 \pm 0.05$ & $1.6 \pm 0.2$ & $2.8 \pm 1$ & $1.9 \pm 0.1$ & - \\
\hline \hline titanium & $38 \pm 3$ & $0.49 \pm 0.05$ & $0.86 \pm 0.08$ & $6.5 \pm 2$ & - & $\begin{array}{c}-0.05 \pm 0.02 ; \\
0.18 \pm 0.02\end{array}$
\end{tabular}

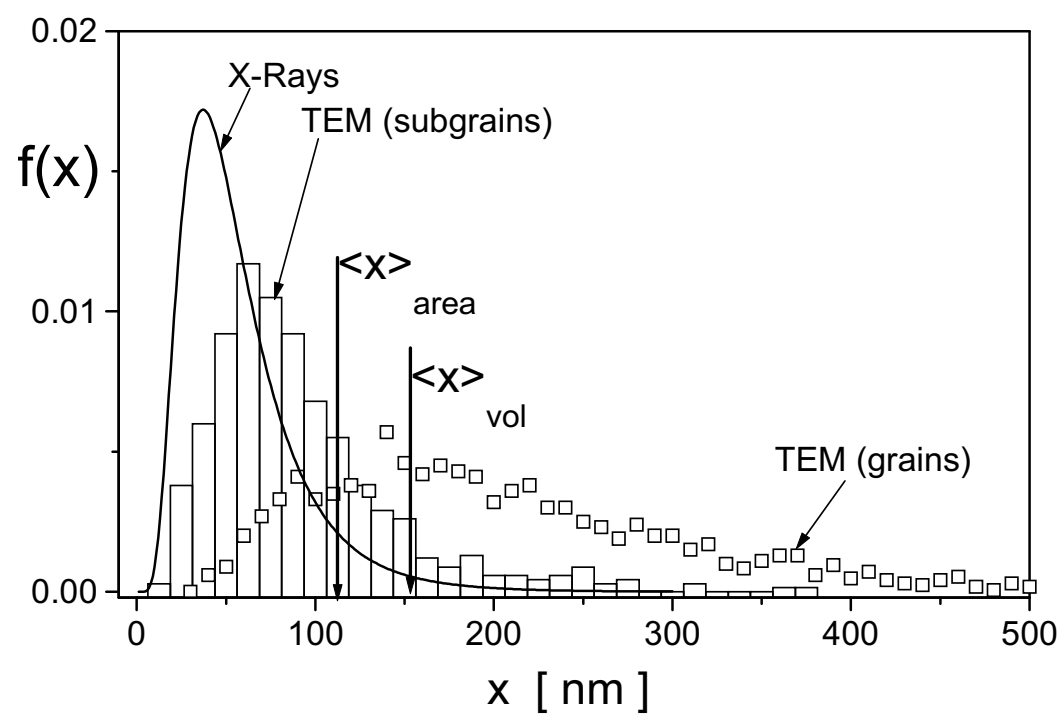

Figure. 2: The size distribution density of grains and subgrains measured by TEM and the size distribution density function of coherently scattering domains or crystallites measured by $X$ rays for ECA pressed copper sample. The area and volume average crystallite sizes, $\langle x\rangle_{\text {area }}$ and $\langle x\rangle_{\text {vol, }}$ respectively, corresponding to the $X$-ray size distribution function, $f(x)$, and defined in eqs. (10) and (11) are also indicated in the figure.

\subsection{The size distribution of grains or subgrains and the dislocation structure in ECA pressed} titanium

The measured and the fitted Fourier coefficients of titanium sample are shown in Fig. 1. The difference between the measured and the fitted values are also shown in the figure. The 
microstructural parameters obtained from X-ray peak profile analysis are listed in Table 1. It can be established that the severe plastic deformation of titanium resulted in nanocrystalline material. The volume- and the area-weighted mean crystallite sizes are 88 and $69 \mathrm{~nm}$, respectively. The $q_{1}$ and $q_{2}$ parameters of the contrast factors depend on the character of dislocations therefore enable the determination of the prevailing dislocation slip systems in the sample. The $q_{1}$ and $q_{2}$ values were calculated for the most common slip systems in hcp metals. Due to the linear superposition of the displacement fields of different dislocations the contrast factors for dislocation population containing two or more slip systems can be calculated as the composition-weighted average of contrast factors of individual slip systems. It can be shown that the experimentally measured $q_{1}$ and $q_{2}$ values in titanium match only the weighted sum of specific pairs of the $q_{1}$ and $q_{2}$ values calculated theoretically for the different slip systems. This is the consequence of the fact that only a few well defined and specific dislocation slip system are activated during deformation [38]. Table 2. shows those calculated $q_{1}$ and $q_{2}$ values which correspond to the active slip systems in Ti. A complete list of the most common slip systems together with the corresponding $q$ factors can be found in [33]. The calculated weighted averages of these three slip systems and the experimental contrast factors for titanium are matched by varying the composition of the dislocation population. A more detailed account of the matching procedure of the theoretical $q_{1}$ and $q_{2}$ values with the meaured data can be found in [39]. As a result of this calculation the following composition of dislocation slip systems was obtained: $67 \pm 5 \%$ (pyramidal edge 4$)+22 \pm 5 \%$ (prismatic edge 1$)+11 \pm 5 \%$ (basal screw). The dominance of the "pyramidal edge 4" system and the negligible presence of the "basal slip" system in titanium is in good agreement with previous TEM observations [38].

Table 2.: The values of $q_{1}$ and $q_{2}$ parameters in the dislocation contrast factors calculated for the three active slip systems in titanium [33].

\begin{tabular}{|cccc|}
\hline \multicolumn{2}{|c}{ slip system } & $q_{1}$ & $q_{2}$ \\
\hline basal screw & $\langle 11 \overline{2} 0\rangle$ & 0.5949 & -0.7104 \\
\hline prismatic edge 1 & $\langle 11 \overline{2} 0\rangle\{1 \overline{1} 00\}$ & -1.1927 & 0.3556 \\
\hline pyramidal edge 4 & $\langle 1 \overline{2} 3\rangle\{11 \overline{2} 1\}$ & 1.5270 & 0.1462 \\
\hline
\end{tabular}

\section{Conclusions}

The crystallite size distribution and the dislocation structure of nanocrystalline copper and titanium produced by severe plastic deformation were studied by X-ray peak profile analysis. The crystallite size distribution obtained from X-rays are in good agreement with the subgrain size distribution determined by TEM. It was found that the dominant dislocation slip system in titanium is "pyramidal edge 4" which correlates with previous TEM observations.

\section{Acknowledgements}

This work was supported by the Hungarian Scientific Research Fund, OTKA, Grant Nos. T031786, T034666 and T029701. J.G. is grateful for the financial support of Magyary Zoltan postdoctoral program of Foundation for Hungarian Higher Education and Research (AMFK). 


\section{References}

1. Langford, J. I., Louër, D. \& Scardi, P. (2000), "Effect of a crystallite size distribution on Xray diffraction line profiles and whole-powder-pattern fitting" J. Appl. Cryst. 33, 964-974.

2. Gubicza, J., Szépvölgyi, J., Mohai, I., Zsoldos L., and Ungár, T., 2000, "Particle size distribution and the dislocation density determined by high resolution X-ray diffraction in nanocrystalline silicon nitride powders", Materials Science and Engineering A280 pp. 263269.

3. Krill, C. E., and Birringer, R., 1998, "Estimating Grain-Size Distribution in Nanocrystalline Materials from X-ray Diffraction Profile Analysis”, Phil. Mag. A, 77, pp. 621-640.

4. Mughrabi, H., 1983, "Dislocation Wall and Cell Structures and Long Range Internal Stresses in Deformed Metal Crystals" Acta metall. 31, pp. 1367-1379.

5. Ungár, T., Mughrabi, H., Rönnpagel, D. and Wilkens, M., 1984, "X-Ray Line Broadening Study of the Dislocation Cell Structure in Deformed [001]-Oriented Copper Single Crystals", Acta Met., 32, pp. 333-342.

6. Gaál, I., 1984, 'Effect of Dislocation Distribution on the X-Ray Scattering from Deformed Metals", in Proc. 5th Riso Int. Symp. on Metallurgy and Material Science, eds. N. Hessel Andersen, M. Eldrup, N. Hansen, D. Juul Jensen, T. Leffers, H. Lilholt, O. B. Pedersen and B. N. Singh, Riso National Lab., Roskilde, Denmark, pp. 249-254.

7. Groma, I., Ungár, T. \& Wilkens, M. 1988, “Asymmetric X-Ray Line Broadening of Plastically Deformed Crystals. Part I: Theory”, J. Appl. Cryst., 21, pp. 47-53.

8. Krivoglaz, M. A., Martynenko, O. V., and Ryaboshapka, K. P., 1983, "Influence of correlation in position of dislocations on X-ray diffraction by deformed crystals",Phys. Met. Metall., 55, pp. 1-12.

9. M. A. Krivoglaz, 1996, in "Theory of X-ray and Thermal Neutron Scattering by real Crystals", Plenum Press, N. Y. 1969; and in X-ray and Neutron Diffraction in Nonideal Crystals, Springer-Verlag, Berlin Heidelberg New York.

10. Wilkens, M. and Bargouth, M.O., 1968, "Die Bestimmung der Versetzungsdichte verformter kupfer Einkristalle aus Verbreiterten Roentgenbeugungsprofilen", Acta metall., 16, pp. 465-468.

11. Wilkens, M., 1970, "The Determination of Density and Distribution of Dislocations in Deformed Single Crystals from Broadened X-Ray Diffraction Profiles", phys. stat. sol. (a) 2, pp. 359-370.

12. M. Wilkens, 1970, "Theoretical Aspects of Kinematical X-ray Diffraction Profiles from Crystals Containing Dislocation Distributions" in Fundamental Aspects of Dislocation Theory, ed. J. A. Simmons, R. de Wit, R. Bullough, Vol. II. Nat. Bur. Stand. (US) Spec. Publ. No. 317, Washington, DC. USA, pp. 1195-1221.

13. Székely, F., Groma, I., and Lendvai. J., 2001, "Statistic Properties of Dislocation Structures Investigated by X-ray Diffraction" Mat. Sci. Eng. A., in the press.

14. Groma, I., 1998, "X-ray Line Broadening Due to an Inhomogeneous Dislocation Distribution" Phys. Rev. B, 57, pp. 7535-1542.

15. Székely, F., Groma, I., and Lendvai. J., 2000, "Characterisation of Self-Similar Dislocation Patterns by X-ray Diffraction" Phys Rev. B, 62, pp. 3093-3098.

16. Wilkens, M., 1988, "X-ray diffraction line broadening and crystal plasticity", Proc. $8^{\text {th }}$ Int. Conf. Strength Met. Alloys (ICSMA 8), Tampere, Finland, eds. P. O. Kettunen, T. K. Lepistö, M. E. Lehtonen, Pergamon Press, pp. 47-152.

17. Williamson, G. K. and Hall, W. H., 1953, "X-Ray Line Broadening from Filed Aluminium and Wolfram", Acta metall., 1, pp. 22-31.

18. Warren, B. E. and Averbach, B. L., 1950, "The Effect of Cold Work Distortions on X-ray Pattern” J. Appl. Phys. 21, pp. 595-610. 
19. Caglioti, G., Paoletti, A. and Ricci, F. P., 1958, "Choice of Collimators for a Crystal Spectrometer for Neutron Diffraction”, Nucl. Instrum. 3, pp. 223-228.

20. Louër, D., Auffredic, J. P., Langford, J. I., Ciosmak, D. and Niepce, J. C., 1983, “A Precise Determination of the Shape, Size and Distribution of Size of Crystallites in Zinc Oxide by X-ray Line Broadening Analysis”, J. Appl. Cryst., 16, pp. 183-191.

21. Klimanek, P. and Kuzel Jr., R., 1988, "X-Ray Diffraction Line Broadening due to Dialocations in Non-Cubic Materials. I. General Considerations and the Case of Elastic Isotropy Applied to Hexagonal Crystals", J. Appl. Cryst. 21, pp. 59-66.

22. Ungár, T. and Borbély, A., 1996, "The effect of dislocation contrast on X-ray line broadening: a new approach to line profile analysis", Appl. Phys. Lett., 69, pp. 3173-3175.

23. Scardi, P. \& Leoni, M. 1999, "Fourier modelling of the anisotropic line broadening of Xray diffraction profiles due to line and plane lattice defects", J. Appl. Cryst. 32, pp. 671682.

24. Cheary, R. W., Dooryhee, E., Lynch, P., Armstrong, N. \& Dligatch, S. 2000, "X-ray diffraction line broadening from thermally deposited gold films", J. Appl. Cryst. 33, pp. 1271-1283.

25. Le Bail, A. \& Jouanneaux, A., 1997, "A Qualitative Account for Anisotropic Broadening in Whole-Powder-Diffraction-Pattern Fitting by Second-Rank Tensors", J. Appl. Cryst. 30, pp. 265-271.

26. Dinnebier, R. E., Von Dreele, R., Stephens, P. W., Jelonek, S. \& Sieler, J. 1999, "Structure of sodium para-hydroxybenzoate, $\mathrm{NaO}_{2} \mathrm{C}-\mathrm{C}_{6} \mathrm{H}_{4} \mathrm{OH}$ by powder diffraction: application of a phenomenological model of anisotropic peak width", J. Appl. Cryst. 32, pp. 761-769.

27. Stephens, P. W., 1999, "Phenomenological Model of Anisotropic Peak Broadening in Powder Diffraction”, J. Appl. Cryst, 32, pp. 281-288.

28. Ungár, T., Dragomir, I., Révész, Á., and Borbély, A., 1999, "The Contrast Factors of Dislocations in Cubic Crystals: the Dislocation Model of Strain Anisotropy in Practice", J. Appl. Cryst. 32, pp. 992-1002.

29. Ungár, T., Gubicza, J., Ribárik G., and Borbély, A., 2001, "Crystallite Size-Distribution and Dislocation Structure Determined by Diffraction Profile Analysis: Principles and Practical Application to Cubic and Hexagonal Crystals", J. Appl. Cryst. 34, 298-310.

30. Ribárik, G., Ungár, T., and Gubicza, J., "MWP-fit: a Program for Multiple Whole Profile Fitting Using Theoretical Functions", J. Appl. Cryst., in the press.

31. Ungár, T. and Tichy, G., 1999, "The Effect of Dislocation Contrast on X-ray Line Profiles in Untextured Polycrystals", Phys. Stat. Sol. (a) 171, pp. 425-434.

32. Hinds, W. C. 1982, Aerosol Technology: Properties, Behavior and Measurement of Airbone Particles, Wiley, New York.

33. Dragomir, I. C. and Ungár, T., J. Appl. Cryst. in preparation.

34. Valiev, R. Z., Ishlamgaliev, R. K. \& Alexandrov, I. V. (2000). Progr. Mater. Sci. 45, 103-189.

35. Valiev, R. Z., Kozlov, E. V., Ivanov, Yu. F., Lian, J., Nazarov, A. A. \& Baudelet, B. (1994). Acta metall. mater. 42, 2467-2476.

36. Stokes, A. R., (1948), "A numerical Fourier-analysis method for the correction of widths and shapes of lines on X-ray diffraction photographs" Proc. Phys. Soc. London, 61, 382-393.

37. Zehetbauer, M. and Seumer, V. (1993),"Cold work hardening in stages Iv and V of FCC metals-I. Experiments and interpretation" Acta metall. mater. 41, 577-588.

38. Jones, I. P. and Hutchinson, W. B., (1981), "Stress-state dependence of slip in Titanium-6Al4V and other HCP metals" Acta Met. 29, 951-968.

39. Zhu, T.Y., Gubicza, J., Dragomir, C. I., Valiev, R. Z. and Ungár, T., Acta Mater., in preparation. 